\title{
LINE-1 hypomethylation in familial and sporadic cancer
}

\author{
Walter Pavicic • Emmi I. Joensuu • Taina Nieminen • \\ Päivi Peltomäki
}

Received: 4 August 2011 /Revised: 6 December 2011 / Accepted: 15 December 2011 / Published online: 8 January 2012

(C) The Author(s) 2012. This article is published with open access at Springerlink.com

\begin{abstract}
Increased and decreased methylation at specific sequences (hypermethylation and hypomethylation, respectively) is characteristic of tumor DNA compared to normal DNA and promotes carcinogenesis in multiple ways including genomic instability. Long interspersed element (LINE), an abundant class of retrotransposons, provides a surrogate marker for global hypomethylation. We developed methylationspecific multiplex ligation-dependent probe amplification assays to study LINE-1 methylation in cases of colorectal, gastric, and endometrial cancer $(N=276)$, stratified by patient category [sporadic; Lynch syndrome (LS); familial colorectal cancer type X (FCCX)] and microsatellite instability status. Within each patient group, LINE-1 showed lower methylation in tumor DNA relative to paired normal DNA and hypomethylation was statistically significant in most cases. Interestingly, normal colorectal mucosa samples from different patient groups displayed differences in LINE-1 methylation that mirrored differences between the respective tumor tissues, with a decreasing trend for LINE-1 methylation from patients with sporadic colorectal cancer to LS to FCCX. Despite the fact that the degree of LINE-1 methylation is generally tissue specific, normal colorectal mucosa, gastric mucosa, and endometrium from LS patients showed similar levels of LINE-1 methylation. Our results suggest that the degree of LINE-1 methylation may constitute a "field defect" that may predispose normal tissues for cancer development.
\end{abstract}

Electronic supplementary material The online version of this article (doi:10.1007/s00109-011-0854-z) contains supplementary material, which is available to authorized users.

W. Pavicic · E. I. Joensuu • T. Nieminen • P. Peltomäki $(\triangle)$ Department of Medical Genetics, Haartman Institute, University of Helsinki,

Helsinki, PO Box 63, 00014, Finland

e-mail: paivi.peltomaki@helsinki.fi
Keywords LINE-1 · DNA methylation · Hereditary cancer · Gastrointestinal cancer - Lynch syndrome

\section{Introduction}

Methylation of cytosine residues at $\mathrm{CpG}$ dinucleotides is a major epigenetic modification in human cells, and $70-80 \%$ of all dinucleotides are heavily methylated [1]. This is true, for example, for transposable elements such as long interspersed elements (LINEs), which constitute $17 \%$ of the human genome being present at over 500,000 copies [2]. Regions that are generally free of methylated $\mathrm{CpG}$ dinucleotides include the so-called $\mathrm{CpG}$ islands, which are around 1-kb stretches of DNA with high GC content enriched in promoter regions of genes [3]. Approximately 27,000 $\mathrm{CpG}$ islands have been identified in the nonrepetitive portion of the human genome [4]. In contrast to normal cells, cancer cells often show reciprocal hypermethylation of $\mathrm{CpG}$ islands $[\mathrm{CpG}$ island methylator phenotype (CIMP)] along with a global decrease in 5methylcytosine content in the remainder of the genome [5].

The full-length LINE-1 element is $6 \mathrm{~kb}$ long and includes molecular machinery that allows retrotransposition. Most LINE-1 copies have become inactive over time as a result of truncations and mutations; some 4,000 remain full length and up to 100 copies may be functional [6, 7]. Ongoing LINE-1 retrotransposition is a prominent source of interindividual genetic variation [7] and an important cause of human genetic disease [8]. In cancer, DNA hypomethylation can result in LINE-1 activation and consequent retrotransposition throughout the genome leading to disruption of genes and chromosomal instability (CIN) [9]. Moreover, an activated LINE-1 promoter can initiate sense or antisense transcription through other genes $[2,6]$. The methylation status of retrotransposons may also contribute to the long- 
range epigenetic control of activity of neighboring genes [10].

Like CpG island methylation, LINE-1 hypomethylation can occur early in tumorigenesis. Ibrahim et al. [11] showed a decrease in LINE-1 methylation from normal mucosa to adenoma (statistically nonsignificant) and from adenoma to carcinoma (significant). Park et al. [12] found a progressive decrease of LINE-1 methylation from chronic gastritis to intestinal metaplasia to gastric adenoma to gastric cancer. Moreover, LINE-1 hypomethylation in tumors may be associated with familial cancer [13, 14], and blood levels of LINE-1 methylation may be associated with increased cancer risk [15] and/or familial cancer [16], suggesting a possible role of LINE-1 methylation in familial or hereditary cancer. Prompted by these findings, we undertook an investigation to examine the levels of LINE-1 methylation in normal and cancer tissues from individuals with familial/ hereditary cancers and their sporadic counterparts, stratified by microsatellite instability (MSI) status. Familial/hereditary cancers included Lynch syndrome (LS), a multi-organ cancer syndrome due to inherited mutations in DNA mismatch (MMR) genes, and familial colorectal cancer, type $\mathrm{X}$ (FCCX), referring to familial nonpolypotic colorectal cancer without MMR defects. For LINE-1 methylation analysis, a novel assay based on methylation-specific multiplex ligation-dependent probe amplification (MS-MLPA) was developed and validated to facilitate studies on formalinfixed paraffin-embedded (FFPE) samples.

\section{Materials and methods}

Patients and samples

LINE-1 methylation was investigated in 276 tumors and corresponding normal tissue samples, consisting of 168 colorectal carcinomas (CRC), 58 gastric carcinomas (GC, 41 of which were intestinal and 17 diffuse), and 50 endometrial carcinomas (EC) (Table 1). All human studies were approved by the appropriate institutional review boards of the Helsinki University Central Hospital. Samples were previously characterized for MSI status and MMR protein expression [17-20]. Germline mutation status stratified the cases into LS (Lynch syndrome with germline mutations in MSH2, MLH1, or MSH6), FCCX (familial nonpolypotic CRC without MMR gene mutations), and sporadic cases (the latter were divided into microsatellite-stable, MSS, and microsatellite-unstable, MSI, subgroups). DNA was extracted from FFPE tumor blocks from selected areas with high tumor percentages and matching normal tissue by a method modified from Isola et al. [21]. Additionally, seven commercial cell lines (HCT116, HCT15, HCA7, RKO, KM12, HEC59, and K-562), one sample from a healthy individual, and one unmethylated control sample, which was generated using a GenomePlex complete whole genome amplification (WGA) kit (Sigma-Aldrich Chemie Gmbh, Germany) [22] were used to compare MS-MLPA and combined bisulfite restriction analysis (COBRA).

\section{Custom-made MS-MLPA for LINE-1}

In MS-MLPA [23], a signal peak is generated if sample DNA is methylated because methylation protects DNAprobe hybrids against digestion by the methylationsensitive enzyme HhaI (cuts at GCGC), and the ligated probes can be amplified by the polymerase chain reaction. For custom-made MS-MLPA design, three areas inside the LINE-1 promoter sequence (with an HhaI site), and seven more regions, located in the remaining portion of the LINE1 element (without HhaI recognition sequence), were chosen, and a specific MS-MLPA probe pair was created for each, giving rise to the so-called "L1-m probes" and "control probes," respectively (see Table 2 and Fig. 1). A published sequence for L1.2 (GenBank accession no. M80343, http://www.ncbi.nlm.nih.gov/nuccore/339773) was used. For the synthetic MS-MLPA probe design, the manufacturer's instructions (http://www.mrc-holland.com) were followed. An MS-MLPA probe pair consists of two oligonucleotides, the left probe oligonucleotide (LPO) and the right probe oligonucleotide (RPO). Custom-made MS-MLPA probes (Table 2), including seven control probes and three methylation-sensitive probes were combined to make a complete MS-MLPA assay (LINE-1-MS-MLPA kit). MS-MLPA reaction was performed using the SALSA MS-MLPA Reagents kit (MRC-Holland, The Netherlands), following a custom-modified MS-MLPA standard reaction protocol. We used 80-110 ng of DNA sample, and the ligation/digestion reaction was performed with $0.75 \mu$ l of HhaI enzyme (Promega $\mathrm{R} 6441,10 \mathrm{U} / \mathrm{\mu l}$ ) for $50 \mathrm{~min}$ at $49^{\circ} \mathrm{C}$. Finally, 24 cycles were used in PCR reaction. All other conditions were as detailed in MRC-Holland instructions.

Methylation dosage ratio was obtained by the following calculation: $D_{\mathrm{m}}=\left(P_{\mathrm{x}} / P_{\text {ctrr }}\right)_{\mathrm{Dig}} /\left(P_{\mathrm{x}} / P_{\text {ctrr }}\right)_{\mathrm{Undig}}$, where $D_{\mathrm{m}}$ is the methylation dosage ratio, $P_{\mathrm{x}}$ is the peak area of the given probe, $P_{\text {ctrl }}$ is the sum of the peak area of all control probes, Dig stands for HhaI digested sample, and Undig stands for the undigested sample. $D_{\mathrm{m}}$ can vary between 0 and 1.0 (corresponding to $0-100 \%$ of methylated DNA).

\section{LINE-1 methylation analysis by COBRA}

LINE-1 promoter methylation was investigated by a modified COBRA PCR protocol reported by Chalitchagorn et al. [24]. DNA was first modified using the CpGenome DNA Modification Kit (Chemicon, Temecula, CA, USA) and the bisulfite converted DNA was subjected to 35 cycles of PCR with two 
Table 1 Description of the study series

\begin{tabular}{|c|c|c|c|c|c|c|c|c|}
\hline & \multicolumn{4}{|c|}{ Colorectal cancer } & \multicolumn{3}{|c|}{ Gastric cancer } & \multirow{2}{*}{$\begin{array}{l}\text { Endometria } \\
\text { cancer } \\
\text { Lynch-EC }\end{array}$} \\
\hline & $\begin{array}{l}\text { Sporadic } \\
\text { CRC MSS }\end{array}$ & $\begin{array}{l}\text { Sporadic } \\
\text { CRC MSI }\end{array}$ & $\begin{array}{l}\text { Lynch- } \\
\text { CRC }\end{array}$ & FCCX & $\begin{array}{l}\text { Sporadic } \\
\text { GC MSS }\end{array}$ & $\begin{array}{l}\text { Sporadic } \\
\text { GC MSI }\end{array}$ & $\begin{array}{l}\text { Lynch- } \\
\text { GC }\end{array}$ & \\
\hline Total number of tumors & 55 & 52 & 43 & 18 & 34 & 11 & 13 & 50 \\
\hline Mean age of diagnosis (years) & 70.3 & 73.5 & 45.7 & 56.4 & 71.1 & 76.5 & 57.5 & 49.9 \\
\hline Germline mutation in MMR genes (total) & N/A & $\mathrm{N} / \mathrm{A}^{\mathrm{a}}$ & 43 & 0 & $\mathrm{~N} / \mathrm{A}$ & $\mathrm{N} / \mathrm{A}^{\mathrm{a}}$ & 13 & 50 \\
\hline$M L H 1$ & & & 38 & & & & 11 & 41 \\
\hline MSH2 & & & 5 & & & & 2 & 4 \\
\hline MSH6 & & & 0 & & & & 0 & 5 \\
\hline MSI-high & 0 & 14 & 40 & 1 & 0 & 11 & 13 & $33^{\mathrm{b}}$ \\
\hline
\end{tabular}

$N / A$ not applicable

${ }^{\text {a }}$ Due to $M L H 1$ promoter methylation in most cases.

${ }^{\mathrm{b}}$ In the same series, MMR protein corresponding to the germline mutation was lost in $36 / 36,100 \%$, cases available for immunohistochemical analysis

primers, 5'-GCGTAAGGGGTTAGGGAGTTTTT-3' and 5'RTAAAACCCTCCRAACCAAATATAAA-3', with an annealing temperature of $50^{\circ} \mathrm{C}$. The amplicons were digested in $15 \mu \mathrm{l}$ reaction volume with $20 \mathrm{U}$ of TaqI or $10 \mathrm{U}$ of TasI in $1 \times$ corresponding enzyme buffer (MBI Fermentas, USA) at $65^{\circ} \mathrm{C}$ for $6 \mathrm{~h}$. The digested PCR products were electrophoresed in 4.5\% NuSieve 3:1 agarose gels (Cambrex, Iowa, USA). Intensities of the digested and undigested bands were obtained by scanning and scoring with NIH Image Software [25]. LINE-1 methylation level was calculated as a percentage of intensity of TaqI divided by the sum of TaqI- and TasI-positive amplicons. The LINE-1 amplicon size is $160 \mathrm{bp}$. Methylated amplicons, TaqI positive, yielded two 80-bp DNA fragments, whereas unmethylated amplicons, TasI-positive, yielded 63- and 97-bp fragments.

\section{Statistical analysis}

Statistical analyses were performed using SPSS Statistics Software (IBM SPSS, Inc., Chicago, IL, USA). Statistical significance for the differences between distributions was evaluated as follows. Depending on whether or not the data were normally distributed (as evaluated by Shapiro-Wilk test), a parametric or nonparametric test, respectively, was chosen. For pairwise analysis of correlated samples (intragroup comparisons), $t$ test (parametric) or Wilcoxon signed rank test (nonparametric) was used. To evaluate the significance of difference between the means of two independent groups (inter-group comparisons), $t$ test (parametric) or Mann-Whitney $U$ test (nonparametric) was applied. For the comparison of multiple $(\geq 3)$ independent groups with normally distributed data, we used one-way ANOVA (or Welch test when the assumption of homogeneity of variances, as evaluated by Levene's test, was not met). This was followed by an appropriate post hoc test (in our case, Games-Howell test as homogeneity of variances was not fulfilled) to determine which particular groups differed. For correlations, the Pearson product-moment correlation coefficient $(r)$ for linear correlation was determined for parametric data and the Spearman rank correlation coefficient (rho) for nonparametric data. $P$ 's $<0.05$ (two-tailed) were considered significant.

\section{Results}

\section{MS-MLPA assay design}

A custom-made MS-MLPA assay was designed as described in "Materials and methods" to monitor the methylation status of three HhaI sites (with the respective probe pairs designated as $1 \mathrm{~m}, 2 \mathrm{~m}$, and $3 \mathrm{~m}$ ) inside the LINE-1 promoter sequence (Fig. 1). Representative images from MS-MLPA analysis are shown in Fig. 2a. $D_{\mathrm{m}}$ values obtained with the three different probe pairs were highly concordant with each other $(P<0.001$ by Pearson correlation analysis for the entire sample series). All results below will be given using $2 \mathrm{~m}$ probes because the same region was included in most assays developed to date for LINE-1 methylation studies (e.g., COBRA assay used by Chalitchagorn et al. [24], and pyrosequencing assay used by Goel et al. [14], see Fig. 1). Evaluation of cell lines and other test samples revealed a good correlation $(P<0.001)$ between $D_{\mathrm{m}}$ values obtained by MS-MLPA and the fraction of methylated LINE-1 sequences obtained by COBRA (Fig. 2b). The same was true for patient specimens (Supplementary Fig. 1). In studies on patient specimens, a whole genome amplified DNA and a cell line DNA with a known $D_{\mathrm{m}}$ value were included in each assay to assess $H$ haI cleavage and reproducibility, respectively. 


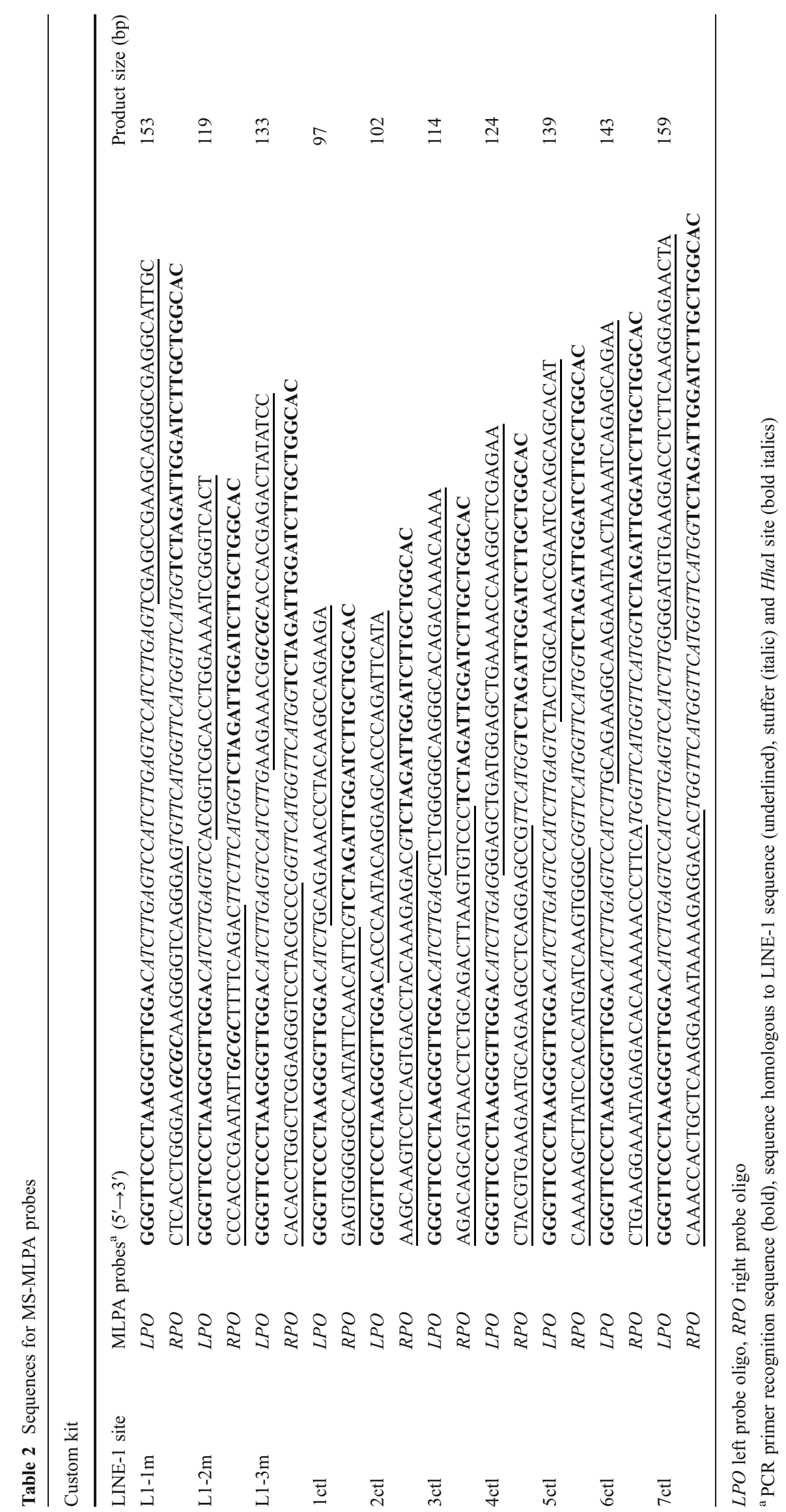




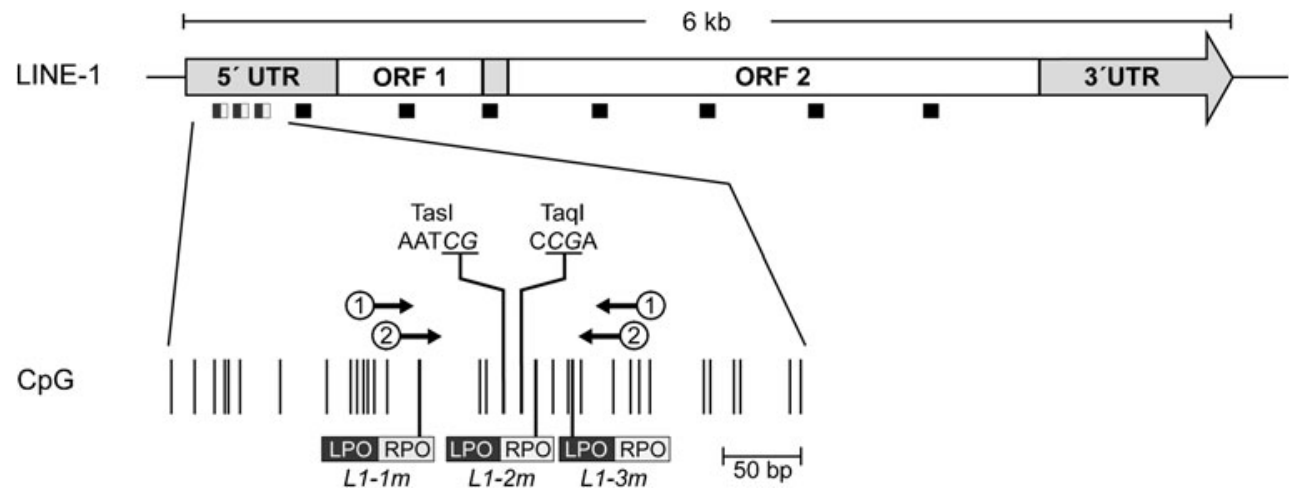

Fig. 1 Diagram of the $\mathrm{CpG}$ island promoter (GenBank accession no. M80343, nucleotide position 108-520 bp) associated with the full length LINE-1. The 5'UTR, 3'UTR and two open reading frames (ORF) of LINE-1 are shown. Location of all ten probe pairs included in the present MS-MLPA assay are indicated below the LINE-1 bar by black/grey and black squares (the so-called L1-m and control probes, respectively). Single $\mathrm{CpG}$ sites in the region containing the L1-m probes are shown by vertical lines in the lower part of the diagram.

LINE-1 methylation in normal vs. tumor tissues: intra-group comparison

We studied 276 cancer specimens and paired normal tissues (representing colorectal mucosa, gastric mucosa, or endometrium) from individuals with familial/hereditary or sporadic cancers, divided into eight groups (Fig. 3, Supplementary Table 1, Supplementary Fig. 2). In all groups, the average LINE-1 methylation dosage ratio was lower in tumor DNA relative to normal DNA, and the degree of hypomethylation reached statistical significance in most groups (denoted by asterisks in Fig. 3). In individuals from whom three different types of tissues were available (Fig. 4, Supplementary Table 2, Supplementary Fig. 3), LINE-1 methylation showed a tissuespecific pattern with the highest level in blood, followed by normal epithelium, and the lowest level in tumors.

LINE-1 methylation in normal and tumor tissues from different patient groups: inter-group comparison

One-way ANOVA analysis of the eight tumor groups shown in Fig. 3 indicated a statistically significant difference between groups $(P<0.001)$. Among CRC tumors, the average LINE-1 methylation dosage ratio (from the highest to the lowest) was 0.87 for sporadic MSI-CRC, 0.85 for sporadic MSS-CRC, 0.84 for LS-CRC, and 0.80 for FCCX (the difference was statistically significant between the first and the last group, $P=$ 0.042 by the Games-Howell post hoc test; Fig. 3 ). The results suggest that LINE-1 methylation in CRCs depended on MSI status and patient category. Among GC tumors, LINE-1 methylation was 0.88 for sporadic MSI-GC, 0.86 for LS-GC, and 0.79 for sporadic MSS-GC (the difference was statistically significant between the first and the last group, $P=0.018$ ).
Numbered arrows indicate the location of primers: 1- used for bisulfite PCR in COBRA assay [in the present work and by Chalitchagorn et al. [24], see "Materials and methods"]; 2- used for pyrosequencing by Goel et al. [14]. The exact CpG sites that are part of HhaI recognition sequences in the three L1-m probes as well as the two CpG sites recognized by restriction enzymes used in COBRA are shown by thick vertical lines. LPO and RPO denote sequences homologous to LINE-1 and correspond to the underlined sequences in Table 2

Furthermore, while LS-GC and sporadic MSI-GC were all of intestinal histology, MSS-GC consisted of tumors with diffuse vs. intestinal histology, and the average LINE-1 methylation was significantly higher in diffuse tumors $(0.85$ vs. $0.74, P=$ 0.005 by two-tailed $t$ test). Our results suggest that MSI status and histology influenced LINE-1 methylation in GC.

There was also a statistically significant difference between the normal tissue groups shown in Fig. 3 ( $P<0.001$ by one-way ANOVA). In particular, normal mucosa tissues from CRC patients showed a declining trend in the average LINE- 1 methylation as follows: sporadic MSS-CRC (0.93), sporadic MSICRC (0.91), LS-CRC (0.90), and FCCX (0.84). The GamesHowell post hoc test indicated a statistically significant difference between FCCX with the lowest LINE-1 methylation in normal mucosa and any other group (at the level of $P<0.05$ or at higher significance levels) and between sporadic MSS-CRC and LS-CRC $(P=0.038)$. The findings suggest that LINE-1 methylation signatures in normal mucosa varied according to the specific groups of CRC patients they represented. Furthermore, LINE-1 methylation was lower in all three tissues (blood, normal mucosa, and tumor) from patients with FCCX compared to the respective tissues from patients with LS-CRC (the difference reached statistical significance between normal mucosa samples, $P=0.018$, by $t$ test, and between blood samples, $P=0.020$, by Mann-Whitney $U$ test; Fig. 4). There was no correlation between LINE-1 methylation in normal mucosa or blood and the age of the patients (data not shown).

LINE-1 methylation vs. tumor suppressor gene methylation

Tumor suppressor gene (TSG) methylation data for the present patient groups were mainly available from our 
Fig. 2 a, LINE-1 CpG island promoter methylation analysis by custom-made MS-MLPA kit. Results from an unmethylated sample (WGA) and a methylated sample (FFPE tumor DNA) are shown. Peaks specific to L1-m probes are indicated by arrowheads and the respective Dm values are boxed. Peaks without a label correspond to control probes. b, Graphical comparison of results from LINE-1 promoter methylation analysis by MS-MLPA (Dm, Y- axis) and COBRA (fraction of methylated LINE-1 sequences, $\mathrm{X}$ - axis) revealed a good correlation as indicated by the Pearson test a
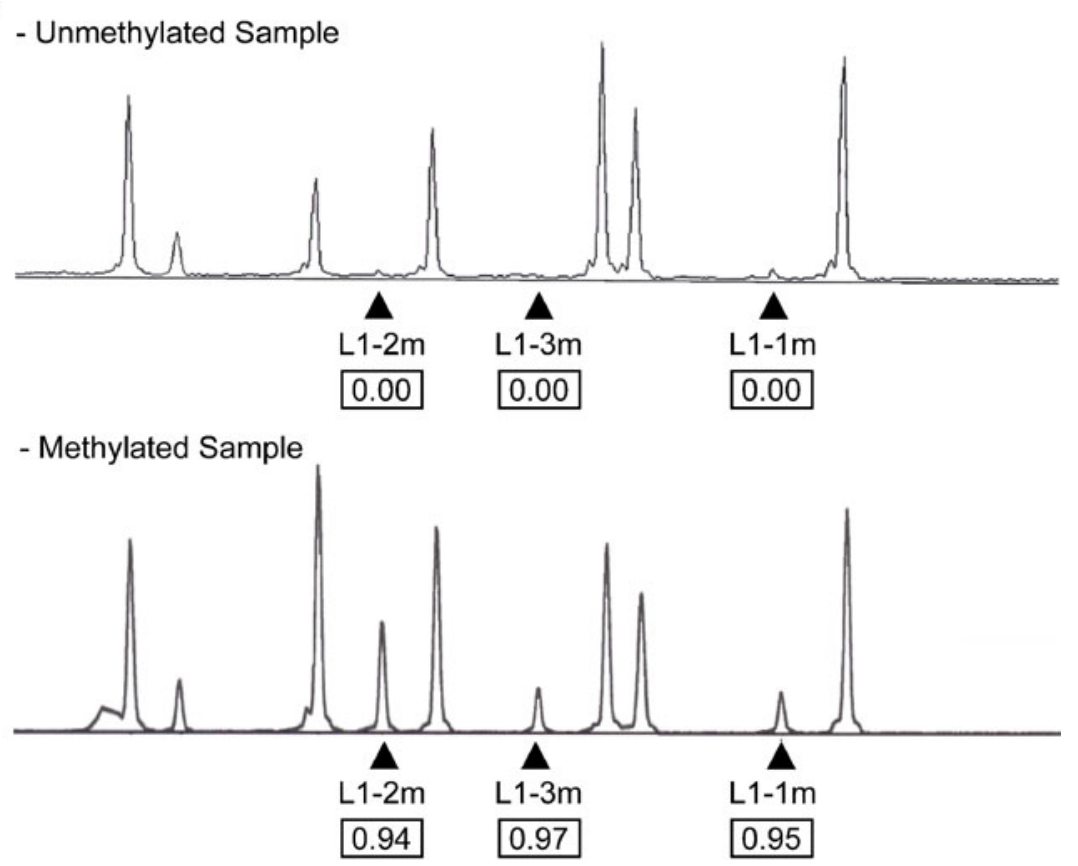

b

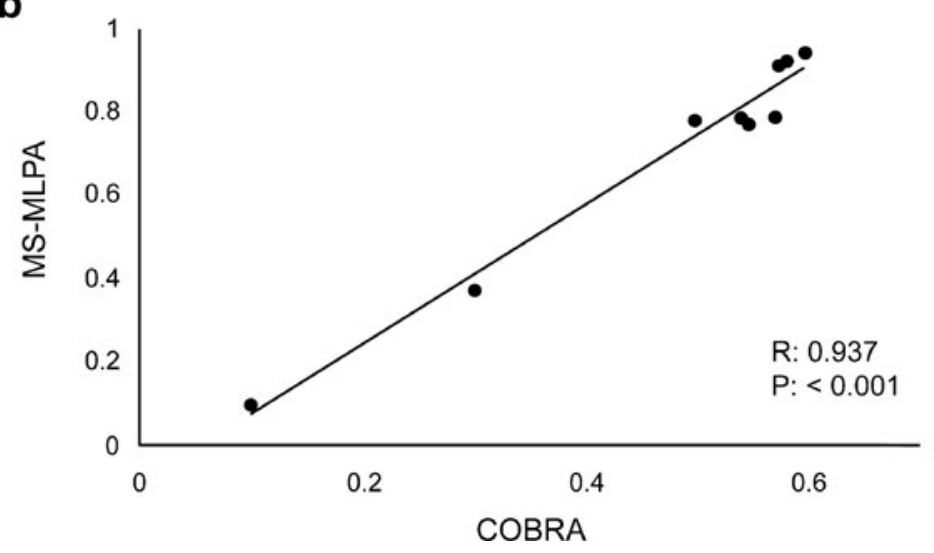

earlier studies, and Supplementary Table 1 gives the average number of methylated TSGs (out of 24 studied) against LINE-1 methylation results. As evident from Supplementary Table 1, LINE-1 methylation was able to distinguish tumor from normal tissue in some groups where TSG methylation could not (e.g., sporadic MSS-GC). In case-by-case analysis by the Pearson test, TSG methylation did not correlate with LINE-1 methylation in tumors from any of the eight patient groups, with a single exception (inverse correlation in LS-EC, $P=0.027$ ).

\section{Discussion}

CRC provides a useful model to study multistep tumorigenesis and the role that DNA methylation plays therein [26]. In sporadic colorectal tumors, genetic instability in the form of CIN or MSI and epigenetic instability in the form of CIMP overlap, resulting in at least three subsets with distinct clinicopathologic features: CIMP+/MSI+, CIMP+/MSI-, CIMP-/CIN [27]. LINE-1 hypomethylation has been reported to be positively associated with CIN $[13,25]$ and inversely with MSI [13, 25, 28, 29]. Among the present sporadic cancers, MSS-CRC and MSS-GC had lower LINE1 values than the respective MSI cancers (the difference was statistically significant among GC) in accordance with reported studies. Variable correlations of LINE-1 hypomethylation with CIMP have been observed in CRC, including inverse association [13, 29] and no association [28]. In our study, LINE-1 hypomethylation did not correlate with TSG methylator phenotype in CRC. Such seemingly inconsistent observations may be reconciled when the basic mechanisms of LINE-1 hypomethylation [30] and CIMP [31, 32], which are largely unknown at present, become better understood.

The overall degree and patterns of DNA methylation tend to be tissue-specific, as shown by studies of $\mathrm{CpG}$ islands 
Fig. 3 Average methylation dosage ratio \pm one standard deviation for LINE-1 in tumor (T) vs. normal $(\mathrm{N})$ tissues. The results are given separately for each patient category out of 8 (listed in the key). Please see Supplementary Table 1 for the exact numerical values and Supplementary Fig. 2 for the relationship between $\mathrm{T}$ and $\mathrm{N}$ case by case. Asterisks indicate statistical significance for the differences between $\mathrm{T}$ and $\mathrm{N}$ within each patient category (intra-group comparison) on the level of $\mathrm{P}<0.05\left(^{*}\right), \mathrm{P}<0.01$ $(* *)$, and $\mathrm{P}<0.001(* * *)$

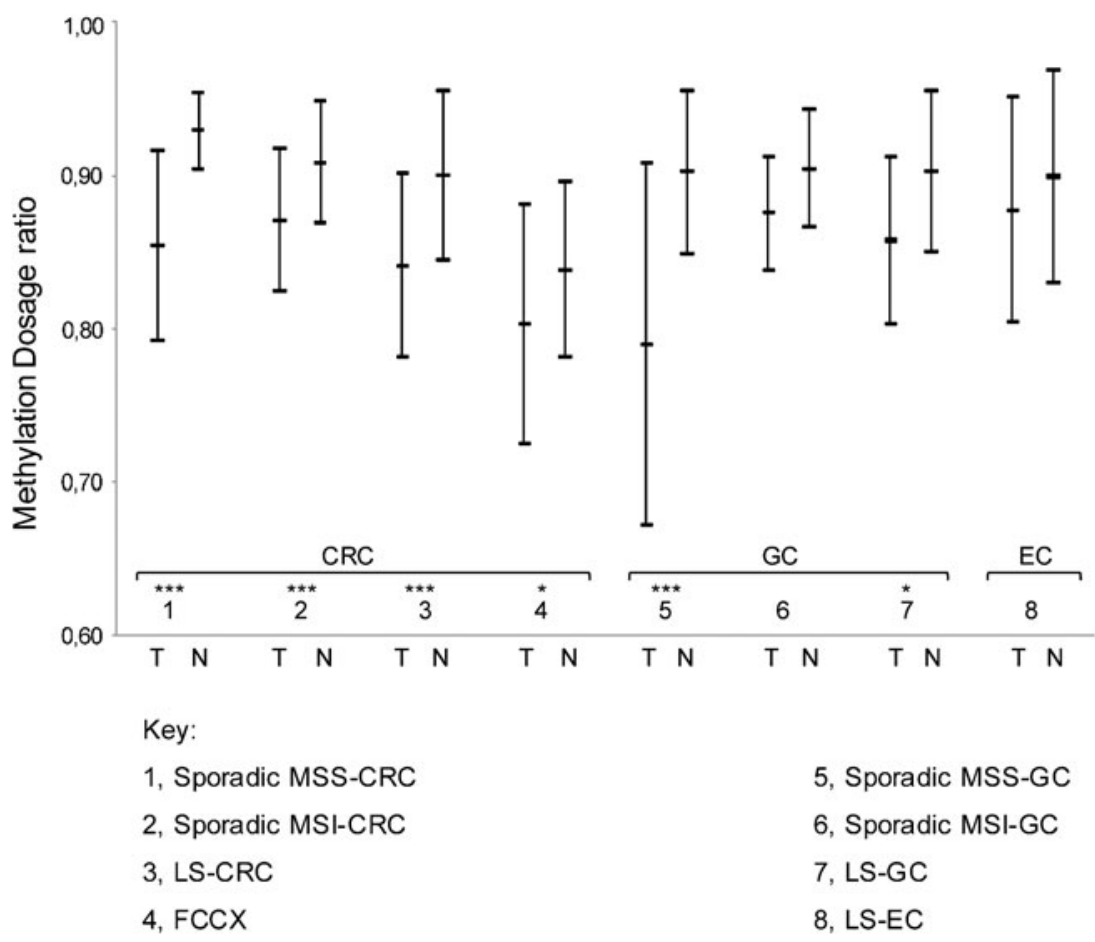

and CpG island "shores" [33], global 5-methylcytosine content [22], and LINE-1 methylation [24]. LINE-1 methylation generally correlates with global genomic 5-methylcytosine content $[22,34]$. An important finding in the present investigation was that even within a given tissue, notably normal colorectal mucosa from patients with $\mathrm{CRC}$, the degree of LINE-1 methylation varied according to the patient category and showed a decreasing trend from sporadic MSS-CRC to sporadic MSI-CRC to LS-CRC to FCCX. A few previous reports exist of DNA methylation in normal colorectal mucosa being associated with pathway-specific predisposition to cancer. Using the MLH1 and CALC1 genes as indicators for methylation, we previously showed that the absence vs. presence of methylation distinguished colonic mucosa in individuals who developed MSI (+) and MSI (-) tumors, respectively [35]. Studies on individuals undergoing colonoscopy for various reasons revealed that age-related ("type A" [36]) methylation in normal mucosa was inversely associated with colorectal adenomas, the precursors of CIN cancers, whereas CIMP ("type C" methylation) was
Fig. 4 Average methylation dosage ratio \pm one standard deviation for LINE-1 in tumor (T) vs. normal colorectal mucosa or endometrium $(\mathrm{N})$ vs. blood (B) from patients with $\mathrm{T}$, $\mathrm{N}$, and $\mathrm{B}$ available. Please see Supplementary Table 2 for the exact numerical values and Supplementary Fig. 3 for the relationship between $\mathrm{T}$ and $\mathrm{N}$ case by case. Asterisks indicate statistical significance for the differences in pairwise comparisons of $\mathrm{T}, \mathrm{N}$, and $\mathrm{B}$ within each patient category (intra-group comparison) on the level of $\mathrm{P}<0.01(* *)$ and $\mathrm{P}<$ $0.001(* * *)$ (-, nonsignificant)

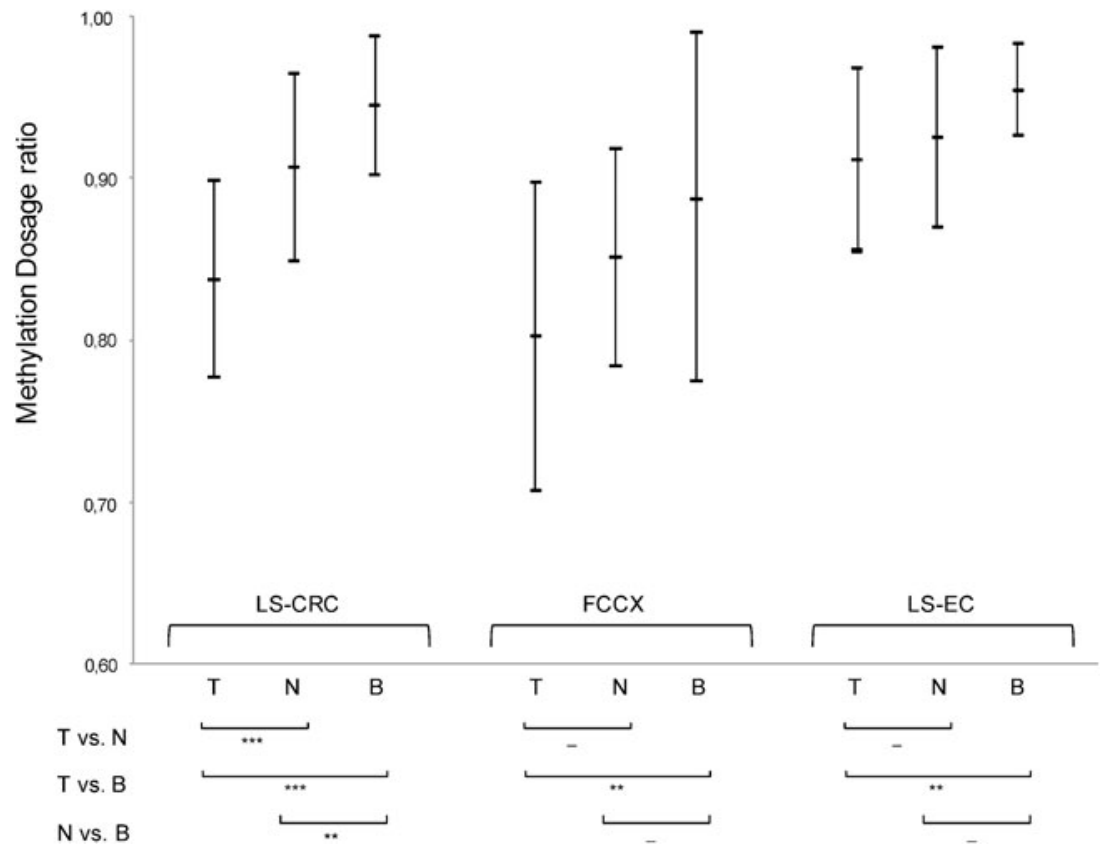


significantly associated with advanced proximal serrated polyps, the precursors of CIMP cancers [37]. However, the latter investigation found no association between LINE-1 methylation status in normal mucosa and subsequent colorectal pathology.

The existing data on LINE-1 hypomethylation in familial/hereditary cancer are scarce. Goel et al. [14] showed that CRCs from FCCX displayed a significantly lower degree of LINE-1 methylation than CRCs from LS or sporadic MSI or MSS cases; results from the corresponding normal tissues were not reported. Among CRCs from our series, too, FCCX showed the lowest mean degree of LINE-1 methylation, further supporting the notion from Goel et al. [14] that LINE-1 methylation is indeed one of the few distinguishing features of FCCX known to date. Nevertheless, the most dramatic differences in our investigation were seen between tissues of normal mucosa from the different patient categories. The patient category seemed to be even more important than tissue type in determining the degree of LINE-1 methylation in normal samples. Apart from the category-specific variation in normal mucosa specimens from CRC patients (see above), all three normal tissues investigated from LS patients (colorectal mucosa, gastric mucosa, and endometrium) and also blood from LS patients with CRC vs. EC showed comparable levels of LINE-1 methylation (Figs. 3 and 4).

FFPE samples stored in pathology archives represent a major source of patient specimens that may remain in part unused because of the lack of methods that take into account the often lower quality and quantity of the extracted DNA. Common methods to study LINE-1 methylation include COBRA [25], MethyLight [22], and pyrosequencing [28]. While these techniques have been applied to FFPE samples as well, all rely on bisulfite conversion and require relatively large amounts of template DNA. We, therefore, took an alternative approach and developed MS-MLPA-based assays that do not require bisulfite conversion, work with limited amounts of template DNA, and allow multiplexed reactions for studies of several regions of LINE-1 simultaneously. While the percentages of LINE-1 methylation reflect the basic technique and the method of calculation used, we demonstrated that our MS-MLPA results were well in line with those obtained with COBRA. Given the increasing evidence of the importance of LINE-1 methylation in cancer and the emerging clinical implications [29], the present assays and findings are likely to stimulate future studies in this field.

Acknowledgments We thank the patients and our clinical and other collaborators for samples. We also thank Saila Saarinen for assisting in laboratory assays. This work was supported by the Academy of Finland grant 121185, the Sigrid Juselius Foundation, the Finnish Cancer Organizations, Biocentrum Helsinki, and European Research Council (FP7-ERC-232635) (to P. P.)
Disclosure The authors declare that they have no competing financial or other interests that might be perceived to influence the results and discussion reported in this paper.

Open Access This article is distributed under the terms of the Creative Commons Attribution Noncommercial License which permits any noncommercial use, distribution, and reproduction in any medium, provided the original author(s) and source are credited.

\section{References}

1. Ehrlich M, Gama-Sosa MA, Huang LH, Midgett RM, Kuo KC, McCune RA, Gehrke C (1982) Amount and distribution of 5methylcytosine in human DNA from different types of tissues of cells. Nucleic Acids Res 10:2709-2721

2. Cordaux R, Batzer MA (2009) The impact of retrotransposons on human genome evolution. Nat Rev Genet 10:691-703

3. Rollins RA, Haghighi F, Edwards JR, Das R, Zhang MQ, Ju J, Bestor TH (2006) Large-scale structure of genomic methylation patterns. Genome Research 16:157-163

4. Han L, Su B, Li WH, Zhao Z (2008) CpG island density and its correlations with genomic features in mammalian genomes. Genome Biology 9:R79

5. Shi H, Wang MX, Caldwell CW (2007) CpG islands: their potential as biomarkers for cancer. Expert Rev Mol Diagn 7:519-531

6. Speek M (2001) Antisense promoter of human L1 retrotransposon drives transcription of adjacent cellular genes. Mol Cell Biol 21:1973-1985

7. Beck CR, Collier P, Macfarlane C, Malig M, Kidd JM, Eichler EE, Badge RM, Moran JV (2010) LINE-1 retrotransposition activity in human genomes. Cell 141:1159-1170

8. Chen JM, Stenson PD, Cooper DN, Ferec C (2005) A systematic analysis of LINE-1 endonuclease-dependent retrotranspositional events causing human genetic disease. Hum Genet 117:411-427

9. Howard G, Eiges R, Gaudet F, Jaenisch R, Eden A (2008) Activation and transposition of endogenous retroviral elements in hypomethylation induced tumors in mice. Oncogene 27:404-408

10. Belancio VP, Deininger PL, Roy-Engel AM (2009) LINE dancing in the human genome: transposable elements and disease. Genome Medicine 1:97

11. Ibrahim AE, Arends MJ, Silva AL, Wyllie AH, Greger L, Ito Y, Vowler SL, Huang TH, Tavaré S, Murrell A et al (2011) Sequential DNA methylation changes are associated with DNMT3B overexpression in colorectal neoplastic progression. Gut 60:499-508

12. Park SY, Yoo E, Cho NY, Kim N, Kang G (2009) Comparison of $\mathrm{CpG}$ island hypermethylation and repetitive DNA hypomethylation in premalignant stages of gastric cancer, stratified for Helicobacter pylori infection. J Pathol 219:410-416

13. Baba Y, Huttenhower C, Nosho K, Tanaka N, Shima K, Hazra A, Schernhammer E, Hunter DJ, Giovannucci EW, Fuchs C et al (2010) Epigenomic diversity of colorectal cancer indicated by LINE-1 methylation in a database of 869 tumors. Molecular Cancer 9:125

14. Goel A, Xicola RM, Nguyen TP, Doyle BJ, Sohn VR, Bandipalliam P, Rozek LS, Reyes J, Cordero C, Balaguer F et al (2010) Aberrant DNA methylation in hereditary nonpolyposis colorectal cancer without mismatch repair deficiency. Gastroenterology 138:1854-1862

15. Ting Hsiung D, Marsit CJ, Houseman EA, Eddy CE, Sloane Furniss C, McClean MD, Kelsey KT (2007) Global DNA methylation level in whole blood as a biomarker in head and neck squamous cell carcinoma. Cancer Epidemiol Biomarkers Prev 16:108-114

16. Mirabello L, Savage SA, Korde L, Gadalla SM, Greene MH (2010) LINE-1 methylation is inherited in familial testicular cancer kindreds. BMC Medical Genetics 11:77 
17. Kuismanen SA, Holmberg MT, Salovaara R, de la Chapelle A, Peltomäki P (2000) Genetic and epigenetic modification of MLH1 accounts for a major share of microsatellite-unstable colorectal cancers. Am J Pathol 156:1773-1779

18. Gylling A, Abdel-Rahman WM, Juhola M, Nuorva K, Hautala E, Järvinen HJ, Mecklin JP, Aarnio M, Peltomäki P (2007) Is gastric cancer part of the tumour spectrum of hereditary non-polyposis colorectal cancer? A molecular genetic study. Gut 56:926-933

19. Joensuu EI, Abdel-Rahman WM, Ollikainen M, Ruosaari S, Knuutila S, Peltomäki P (2008) Epigenetic signatures of familial cancer are characteristic of tumor type and family category. Cancer Res 68:4597-4605

20. Nieminen TT, Gylling A, Abdel-Rahman WM, Nuorva K, Aarnio M, Renkonen-Sinisalo L, Järvinen HJ, Mecklin JP, Bützow R, Peltomäki P (2009) Molecular analysis of endometrial tumorigenesis: importance of complex hyperplasia regardless of atypia. Clin Cancer Res 15:5772-5783

21. Isola J, DeVries S, Chu L, Ghazvini S, Waldman F (1994) Analysis of changes in DNA sequence copy number by comparative genomic hybridization in archival paraffin-embedded tumor samples. Am J Pathol 145:1301-1308

22. Weisenberger DJ, Campan M, Long TI, Kim M, Woods C, Fiala E, Ehrlich M, Laird PW (2005) Analysis of repetitive element DNA methylation by MethyLight. Nucleic Acids Res 33:6823-6836

23. Nygren AO, Ameziane N, Duarte HM, Vijzelaar RN, Waisfisz Q, Hess CJ, Schouten JP, Errami A (2005) Methylation-specific MLPA (MS-MLPA): simultaneous detection of CpG methylation and copy number changes of up to 40 sequences. Nucleic Acids Research 33:e128

24. Chalitchagorn K, Shuangshoti S, Hourpai N, Kongruttanachok N, Tangkijvanich P, Thong-ngam D, Voravud N, Sriuranpong V, Mutirangura A (2004) Distinctive pattern of LINE-1 methylation level in normal tissues and the association with carcinogenesis. Oncogene 23:8841-8846

25. Matsuzaki K, Deng G, Tanaka H, Kakar S, Miura S, Kim YS (2005) The relationship between global methylation level, loss of heterozygosity, and microsatellite instability in sporadic colorectal cancer. Clin Cancer Res 11:8564-8569
26. Davies RJ, Miller R, Coleman N (2005) Colorectal cancer screening: prospects for molecular stool analysis. Nat Rev Can 5:199-209

27. Issa JP (2008) Colon cancer: it's CIN or CIMP. Clin Cancer Res 14:5939-5940

28. Estécio M, Gharibyan V, Shen L, Ibrahim A, Doshi K, He R, Jelinek J, Yang A, Yan P, Huang T et al (2007) LINE-1 hypomethylation in cancer is highly variable and inversely correlated with microsatellite instability. PLoS ONE 2:e399

29. Ogino S, Kawasaki T, Nosho K, Ohnishi M, Suemoto Y, Kirkner GJ, Fuchs CS (2008) LINE-1 hypomethylation is inversely associated with microsatellite instability and $\mathrm{CpG}$ island methylator phenotype in colorectal cancer. Int J Cancer 122:2767-2773

30. Pogribny IP, Beland FA (2009) DNA hypomethylation in the origin and pathogenesis of human diseases. CMLS 66:2249-2261

31. Issa JP (2004) CpG island methylator phenotype in cancer. Nat Rev Cancer 4:988-993

32. McCabe MT, Brandes JC, Vertino PM (2009) Cancer DNA methylation: molecular mechanisms and clinical implications. Clin Cancer Res 15:3927-3937

33. Irizarry $\mathrm{R}$, Ladd-Acosta $\mathrm{C}$, Wen $\mathrm{B}, \mathrm{Wu} \mathrm{Z}$, Montano $\mathrm{C}$, Onyango $\mathrm{P}$, Cui H, Gabo K, Rongione M, Webster M et al (2009) The human colon cancer methylome shows similar hypo- and hypermethylation at conserved tissue-specific $\mathrm{CpG}$ island shores. Nature Genetics 41:178-186

34. Yang AS, Doshi KD, Choi SW, Mason JB, Mannari RK, Gharybian V, Luna R, Rashid A, Shen L, Estecio MR et al (2006) DNA methylation changes after 5-Aza-2'-deoxycytidine therapy in patients with leukemia. Cancer Res 66:5495-5503

35. Kuismanen SA, Holmberg MT, Salovaara R, Schweizer P, Aaltonen LA, de la Chapelle A, Nyström-Lahti M, Peltomäki P (1999) Epigenetic phenotypes distinguish microsatellite-stable and -unstable colorectal cancers. PNAS 96:12661-12666

36. Toyota M, Ahuja N, Ohe-Toyota M, Herman JG, Baylin SB, Issa JP (1999) CpG island methylator phenotype in colorectal cancer. PNAS 96:8681-8686

37. Worthley DL, Whitehall V, Buttenshaw RL, Irahara N, Greco SA, Ramsnes I, Mallitt K-A, Le Leu RK, Winter J, Hu Y et al (2010) DNA methylation within the normal colorectal mucosa is associated with pathway-specific predisposition to cancer. Oncogene 29:1653-1662 